$\mathbb{T}$ periodica polytechnica

Transportation Engineering $38 / 2(2010) 105,111$

doi: $10.3311 /$ pp.tr.2010-2.08

web: http://www.pp.bme.hu/tr

(c) Periodica Polytechnica 2010

RESEARCH ARTICLE

\section{The extraction of unique velocity processes from a macro model}

\author{
Zsuzsanna Bede / Tamás Péter
}

Received 2009-11-03

\begin{abstract}
In our article measured, we compare the velocity profiles received of the detailed traffic simulation modelled by us in the traffic, as EUDC standard procession cycles employed with the introduction of regulations the EURO3. In the course of this, EUDC principal deficiencies turned into conspicuous visible one. The differences already perceptible in case of values which can be counted directly from the velocity profile. The average speed and the time filled in the neutral gear, in the stand are like this for example. The statistical values calculated by our model reflect it well the (measured) sudden acceleration processes typical of traffic, as according to the real processes, and not contain permanently static velocity values. Based on this, we make a proposal - the NEDC cycle different - the application of new velocity profiles. This give much more accurate procedure on the testing of the vehicles to the real procession characteristics of the vehicle the definition of values.
\end{abstract}

\section{Keywords}

non-linear network model $\cdot$ traffic simulation model $\cdot$ extraction of unique velocity processes

\section{Acknowledgement}

This work is connected to the scientific program of the "Development of quality-oriented and harmonized $R+D+I$ strategy and functional model at BME" project. This project is supported by the New Hungary Development Plan (Project ID: TÁMOP4.2.1/B-09/1/KMR-2010-0002).

The presented research matter is sponsored by OTKA CNK 78168 project.

\section{Zsuzsanna Bede}

Department of Control and Transport Automation, BME, Bertalan L. u. 2., H1111 Budapest, Hungary

e-mail: bede.zsuzsanna@mail.bme.hu

\section{Tamás Péter}

Department of Control and Transport Automation, BME, Bertalan L. u. 2., H1111 Budapest, Hungary

e-mail: tamas.peter@mail.bme.hu

\section{Introduction}

They limit the environmental pollution of the vehicle engines with official regulations. The official regulations on vehicle category contain the different vehicle brake test bench or engine brake test bench analytical programs, different measurement procedures and different limit values. The requirements mostly are established according to the peculiarities of the traffic from statistical data [1,2].

The regulations may concern:

- Onto the harmful components of the exhaust gases

- Onto the durability of the values checked on the type examination

- The vehicles may not exceed the one and a half times value of the type examination limitation until the accomplishment of a particular road. (In Europe at the automobiles this 100.000 $\mathrm{km}$.)

- Onto the consumption of the vehicle

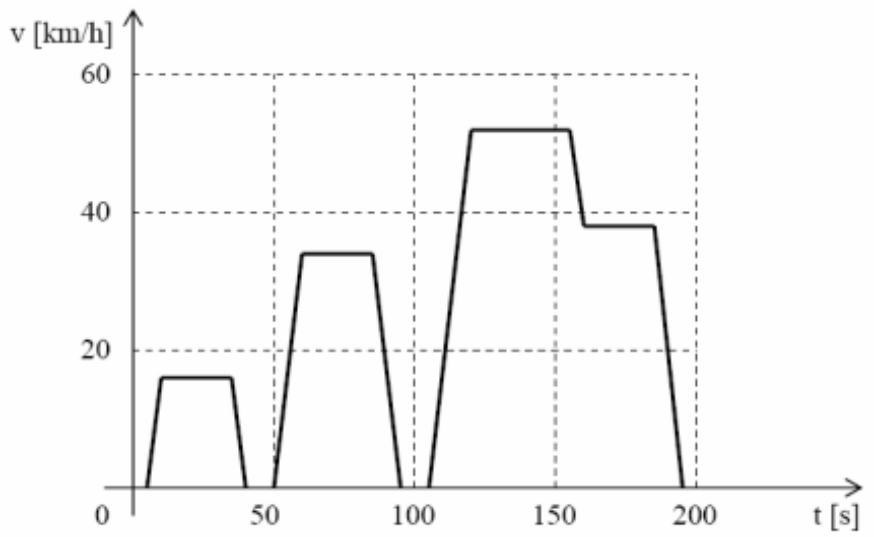

Fig. 1. UDC - Urban Driving Cycle

The progressing profile consists of the series of complicated, complex accelerations, decelerations and frequent stops in the reality $[2,-4]$. These values differ in the laboratory on the vehicle brake bench trundle of measured values. The new European driving cycle (NEDC - New European Driving Cycle, NEFZ - Neue Europäische Fahrzyklus) are applies laboratory 
tests approved in EU, which the two European capitals (Paris and Rome) he was established based on his traffic data.

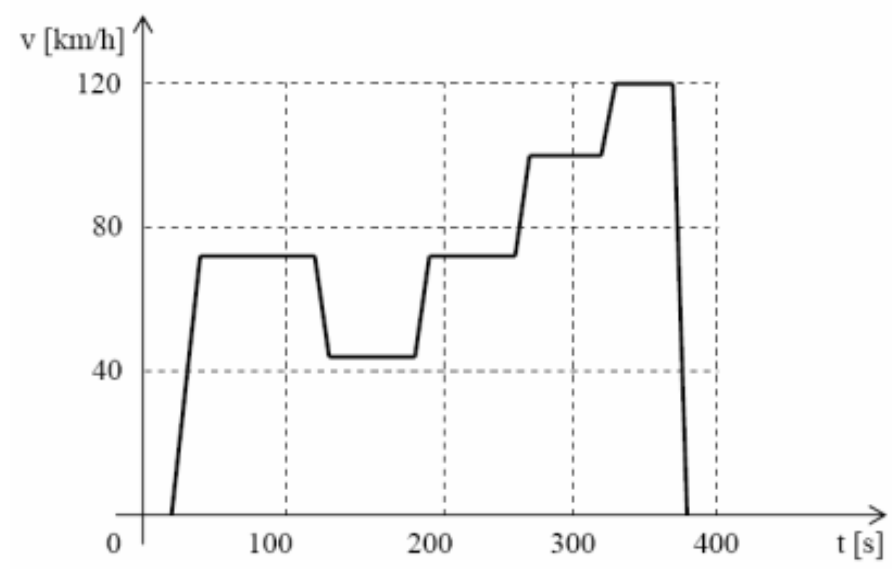

Fig. 2. EUDC - Extra Urban Driving Cycle

The urban European procession cycle is equal to the left side of the diagram which can be seen on the Fig. 3 (UDC - Urban Driving Cycle). The disadvantage of this, that leave out of consideration the made kilometers on the high road, respectively on a motorway, which expose considerable proportion to the life of many vehicles.

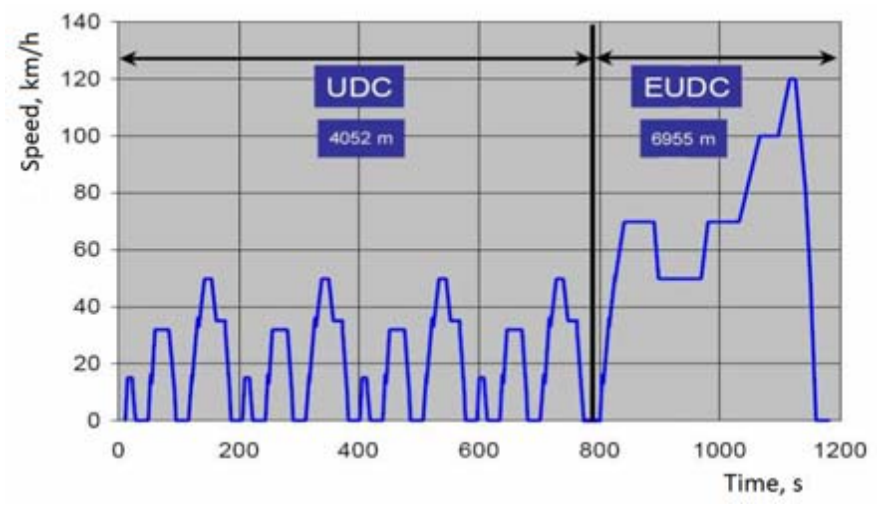

Fig. 3. Urban and apart from a city European driving cycle

With the introduction of regulations EURO3, from 2000 the urban cycle was complemented with a roadside procession cycle (EUDC). A sample is taken from the exhaust gases continuously, component emitted under the full cycle then $\mathrm{g} / \mathrm{km}$ it is converted into a unit. The full cycle was get a name the new European Driving Cycle (NEDC $=$ UDC+EUDC), that consists of 4 urban and 1 roadside cycle: NEDC $=4 \mathrm{UDC}+\operatorname{EUDC}($ Fig. 3 ).

The vehicles emission particularly depends on the style of driving, on which has a big result the conformation of the momentary traffic. The diagrams of velocity-time of the procession cycles represent the urban progress. It is examined with a brake bench measurement the consumption of combustible, the combination of the exhaust gas and his coefficients, considering the conditions of the urban traffic between laboratory circumstances.

The development of the procession cycles we distinguish a way of two kinds. One of the methods alternates the constant fast, deceleration and constant velocity value, so for example NEDC and ECE procession cycles, these cycles reckon it among the polygonal models. The other type already stands near to the real velocity profile, like these real-word - natural world procession cycles reckon among it. Procession cycle like this FTP-75, which is more dynamic compared to the polygonal models, it takes the sudden accelerations and decelerations into consideration, so reflects better the velocity values measured according to the road conditions. This more dynamic velocity profile according to the reality, gives more accurate emission values, than the values measured in the course of tests at which the regular procession diagram is.

The FTP-72 (Federal Test Procedure) driving cycle simulate urban traffic (Fig. 4). The cycle consists of two parts: $505 \mathrm{sec}$ ond and 864 second sections. The first section starts with a cold startup. At the second section the measurement is broken a neutral gear lasting until 10 seconds.

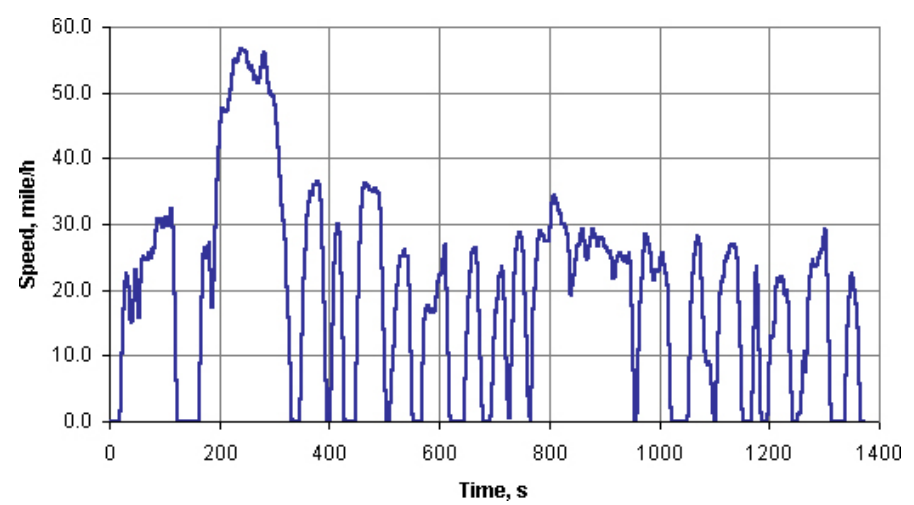

Fig. 4. FTP-72 driving cycles

The FTP-75 procession cycles spring from the FTP-72 procession cycle. The FTP-72 procession cycle are added to a third 505 second sections Fig. 5, what tallies with the first section of the FTP-72 procession cycles, but this is made with a warm neutral gear already. Precedes the third section likewise one 10 minute neutral gear for the engine.

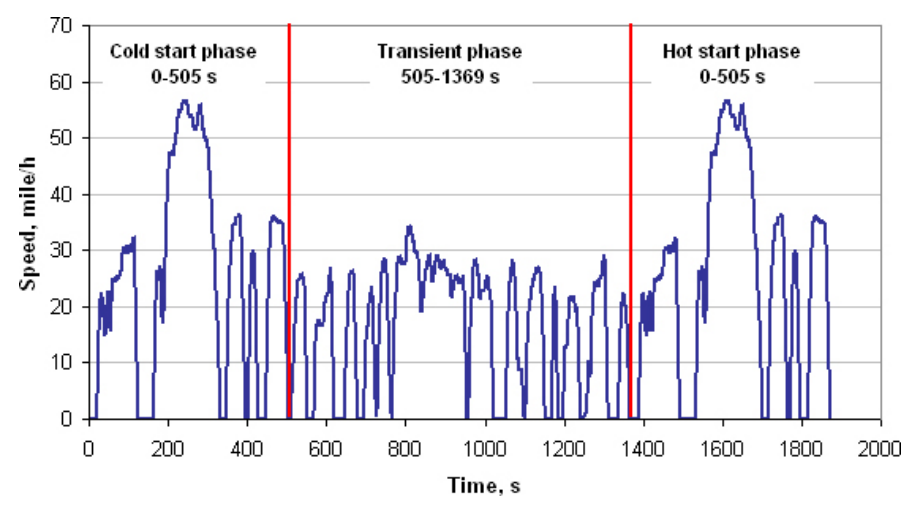

Fig. 5. FTP-75 procession cycles

The exhaust gas is gathered into a separated Teflon bag separately at all three sections, in the course of the evaluation it is expressed in a unit $\mathrm{g} / \mathrm{km}$ ( $\mathrm{g} / \mathrm{mile})$. The $\mathrm{SC03}$ is an accessory test, which shows beside neutral gear the emission of a surplus of vehicles with a unit of air conditioner. 


\subsection{The selection of the procession cycles}

In Europe and like this in Hungary, onto automobiles concerns NEDC procession cycle, that provides information on the behaviour of the vehicle big velocity, and gives an overall picture about the emission. We look at it in anyway, this a skimpy comparison basis the real combustible from consumption and emission. If we examine only capitals of Europe, we may observe considerable differences on these cities considering the establishment of traffic. The factors influencing the traffic among other things the infrastructural forming, the road network and example the given city's public transport system too, which has an effect on the establishment of the stocks of vehicle. Like this, if we would like to receive a real picture in a given city from the effect on environment of a travelling vehicle, so in the course of the measurement has to take the peculiarities the city of this into consideration, indeed, the given country's traffic habits.

\section{Traffic simulation}

We consider the traffic model and the simulation program build on this, which program is developed software onto a large measuring road traffic network [5, 6]. A non-linear network model determine his function, which of the contact matrix is built on the under mentioned:

Our traffic network model composed of the $n$ part of inside road-section describe the road/urban traffic system, that seat oneself in a province of delimited with a curve closed [7,8] . In this case the vehicle densities taking shape on an inner network $\left(\mathrm{N}_{i}\right)$ are the state features of the system, by order of succession $\mathrm{x}_{1}(\mathrm{t}), \mathrm{x}_{2}(\mathrm{t}), \mathrm{x}_{3}(\mathrm{t}), \ldots, \mathrm{x}_{n}(\mathrm{t})$. The model, uses that part network of the exterior network $\left(\mathrm{N}_{e}\right)$, that like composed of the $\mathrm{m}$ part of a section, which has a relation direct with one of the inner sections. On these marks the vehicle densities taking shape $s_{1}(t)$, $\mathrm{s}_{2}(\mathrm{t}), \ldots, \mathrm{s}_{m}(\mathrm{t})$, that based on measurements know.

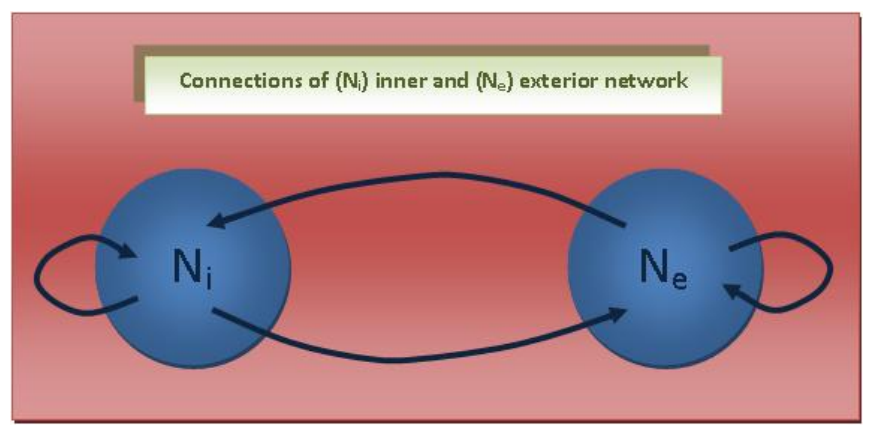

Fig. 6. The contacts of the inner and the exterior network [5]

Our mathematical model descriptive the network takes it into consideration the network inner a province inside and the network exterior a province outside too (Fig. 6). So to creating the network mathematical model the connection matrix defining the network has of fundamental importance, that a hipermatrix.

Our model applies three connection matrices: inside, input and output connection matrix.

The construction of the connection matrices is identical. The

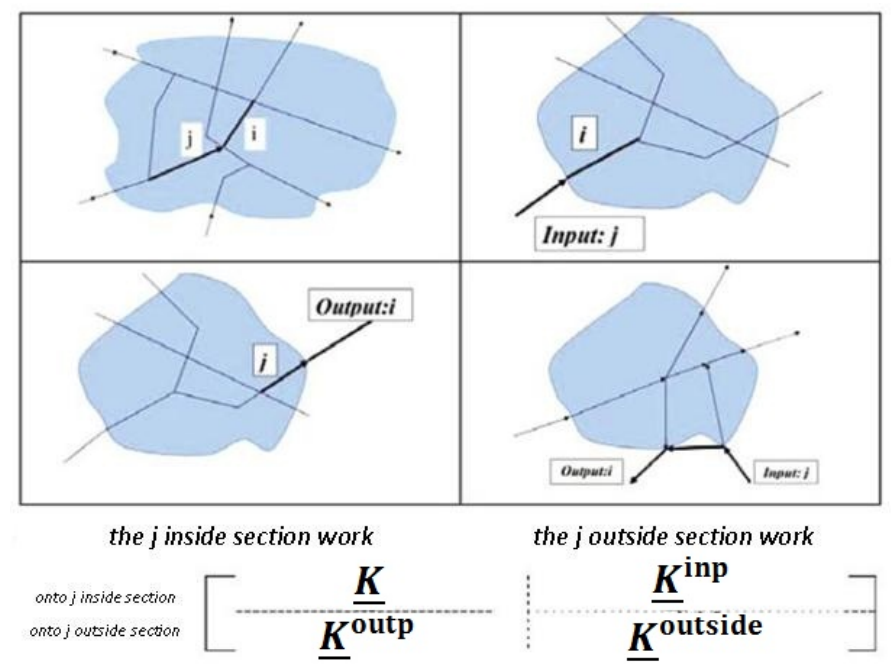

Fig. 7. The inside and the outside network of the connection hipermatrix $|8|$

$i^{\text {th }}$ line of $j$ column we write the $K_{i j}$ connection function $(i \neq \mathrm{j}$, $1 \leq i, j \leq n)$, if the $j$ section is attached the $i$ section. The $K_{i j}$ connection function of the connection matrix it is necessary to take it into consideration on all of them, the forming of the traffic order determined connection feature of regulation (example the connections of a lamp or a without lamp, a road-section - a parking, etc.), we describe these with the $k_{i j}(\mathrm{t})$ function.

You must allow for out of this, that occur inner automatisms of regulation at the time of the formation of the traffic! Our model, we took the inner regulations depending on the density of the traffic into consideration with the application of the $S_{i}(\mathrm{t})$, $E_{j}(\mathrm{t})$ and $v_{i j}(\mathrm{t})$ functions. So the $K_{i j}$ is defined four factors.

- The value of the $k_{i j}(\mathrm{t})$ function, if there is a lamp it take on 1 , 0 values according to the state of the lamp. If there is a constant contact without a lamp and the $j$ section works only $i$ section, then constant 1 is his value, constant 0 is this if there is not a geometry contact between the two sections. If the $j^{\text {th }}$ section works onto more sections without a lamp, then it take down a $0\left\langle\alpha_{i j}\langle 1\right.$ distribution proportion, where in a column $\Sigma(j) \alpha_{i j}=1$. If the contact is disturbed example a crossing vehicles, a pedestrian or an accident, then it take down a value of $0\left\langle\beta_{i j}<1\right.$ disturbing factor. If the contact is helped example a vehicles opposing an other direction or a policeman, then it take down a value of $1+\beta_{i j}$ helping factor. If there are a present distribution and disturbing simultaneously, respectively distribution and helping in, then it occurs a $\alpha_{i j} \beta_{i j}$ resp. a $\alpha_{i j}\left(1+\beta_{i j}\right)$ product.

- The $S_{i}(\mathrm{t})$ automatic inner self-regulation function take down 1,0 values. The permission of a contact, if the density of $i^{t h}$ section $\mathrm{s}_{i}(\mathrm{t})$ smaller, than 1 , anyway 0 .

- The $E_{j}(\mathrm{t})$ automatic inner self-regulation function take down 1,0 values. The prohibition of a contact, if the density of $j^{t h}$ section $s_{j}(\mathrm{t})$ smaller, than 0 , anyway 1 .

- The $v_{i j}(\mathrm{t})$ is the velocity of crossing occurring from the $\mathrm{j}^{\text {th }}$ 
section onto the $\mathrm{i}^{\text {th }}$ section, which is the function of the densities of the joining sections, $v_{i j}(\mathrm{t})=\mathrm{f}\left(s_{i}(\mathrm{t}), s_{j}(\mathrm{t})\right)$.

\section{Non-linear network model is onto the large traffic net- works modeling}

The $x$ vector of a state feature of the non-linear traffic network system can be written down the under mentioned differential equation system compacter shaped:

$$
x_{(n x 1)}^{\prime}=\left\langle 1 / l_{i}\right\rangle_{(n \times n)}\left[K_{(n \times n)} x_{(n \times 1)}+K_{(n \times m)}^{i n p} s_{(m \times 1)}\right]
$$

Where: $K$ and $K^{i n p}$ are the elements of connection matrices, it imply the connection functions and the functions depending on the density states.

The model apply to an entirely new network graph compared to models with a similar topic [6]: the network's real creating elements are the lane sections, so sections cooperate actually with sections in the whole network and these elements constitute the peaks of the network graph. The edges of the directed graph are state pendant dynamic relations, namely in the contact standing, contacts between peaks (cooperating) are dynamic. (The cooperation may be passing, resp. influencing character.) So this model, it placed the network in the central place and examines all of the network beside his full connection system! In this the junction does not appear as an independent element already, namely the function of all junctions is the part of the full connection system! His big strength for the model, that with this technique can be modelled the optional measuring networks.

In the examined province, we number all distances to be taken into consideration on the based of the map. Numbering the edges of the graph, fix of his data geometry and contact happens with an operation of mouse and these data get into a file. We calculate automatically the sections' length and the vehicle numbers which can be displayed maximally on the sections onto a unit vehicle calculated at the same time. Our mathematical model writing down the network is a positive dynamic system. The network takes it into consideration the network inner a province inside and the network outside a province exterior too.

The software - with networks modelling - provides help in the analysis of road traffic vehicle flow processes happening on networks, more ever effect study expanding on the whole network can be prepared in a planning section already. Can be simulated traffic accidents, detours, traffic order changes, a programming changes of traffic light, effect of institute or stopping of a car parks, furthermore expanding occurring of the network by a new road-section, the widening of some road-section, building a roundabout, etc. The peculiarity of the program, that the planning of the road network, the program making the simulation and the surfaces suitable for analysis, it unites, like these are some elements of the adopted network are optionally modifiable, resp. the network expandable too.

\section{Model uptake}

Our examination in Budapest, extends in the boulevard from the Petőfi híd to the Nyugati tér, setting off for a northern direction. The inner sections are create this full length of the boulevard, the inputs and the outputs are side streets working into this section.

The program marks the input section, the output section and the inner section with different colored arrows. Giving of the contact matrix happens to the configuration of the crossroads, a circle marks these crossroads. All of the junctions got to uptake, and we developed crossroads with 40 metres before these junctions, where we can grant parallel passing for the possibility drive down in the junction following. Our examination are aimed the traffic everyday in the morning peak and in the morning.

The site got to ingress, of which video recording was made, and a traffic census happened in the three largest junctions affecting the route, in the junction Ferenc körút - Üllői út, in the Blaha Lujza tér and in the Octogon. Granting of the input functions happened on the basis of measured results.

The parameter of input functions: The model setting the specific quarter is necessary in any case, without the change in the code we practise scale and the input data is approached to the measurement results [5]. Because of this, we may take unique functions, which we may assign to the same input or output sections, to the parking furthermore.

In the course of the day it can be observed protruding traffic increase three times on an average road network; one in the breakfast resp. afternoon peak time, and one is smaller in around the lunch-time. Taking this characteristic into consideration the program offers a constant curve density, in which certain parameters can be made change by way of the user (in time and intensity equally). With the change of these parameters, we put on the density functions conforming to our route which can be seen on the Fig. 9. On this figure the input function of prime section we put two input sections of Petôfi híd, respectively input function of the side streets giving different weighting to the input section on the side street.

The input density function may taken a value between 0 and 1 , that is given section's density is 1 , if the vehicles occupy the given section totally, namely the cars stand from bumper to bumper. In consequence the 0 values mean not vehicle altogether on that given section.

Based on the values measured in the three junctions verifiable, that the considerable part of the traffic current progresses on the designate route, that is onto this section from carrying measure of the side streets is equal onto the side streets from the leaving measure of this section. The simulation correct running justifies, that the running-time measured in the course of the ingress of the site was equal to the running-time measured with of the simulation, according to which it is possible to go along the full route under 25 minutes before 9 clocks. 


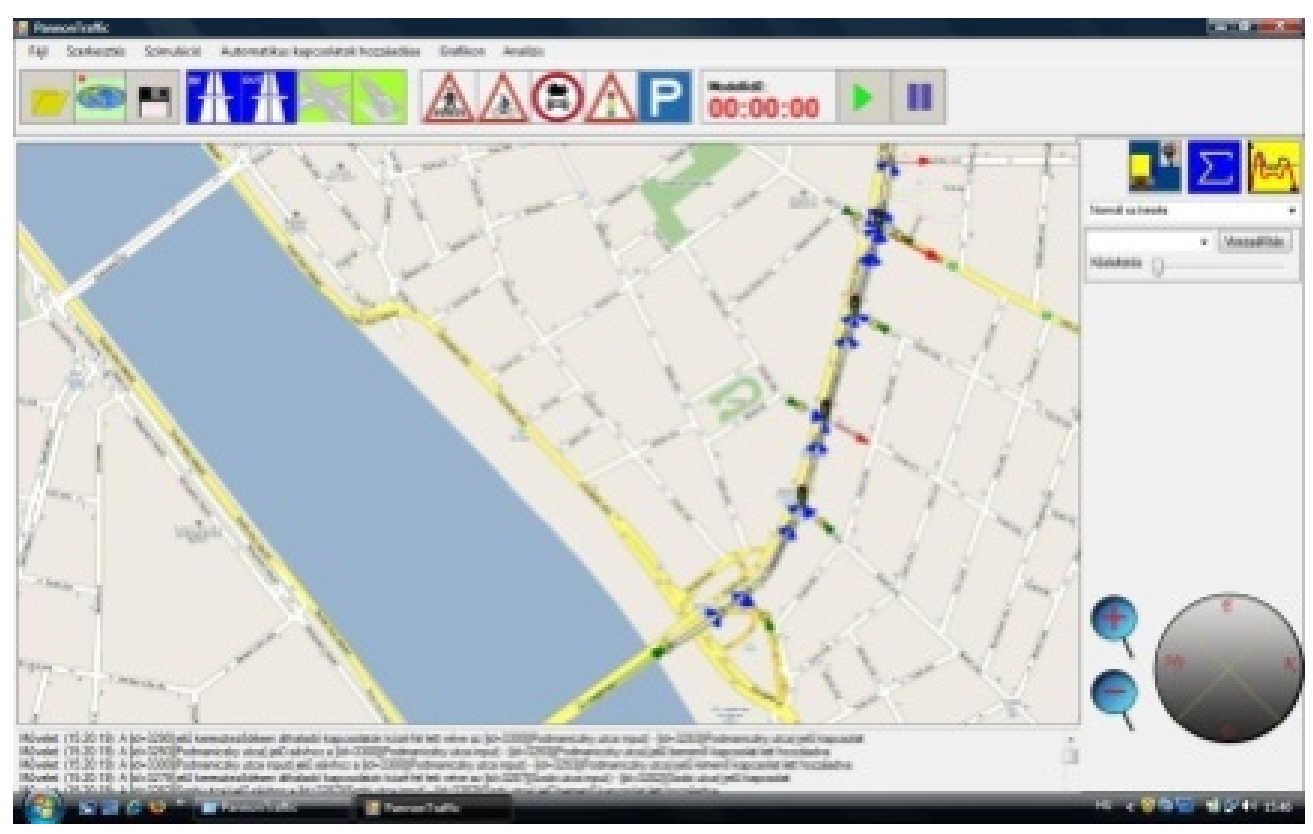

Fig. 8. The window of a Pannon Traffic simulation program
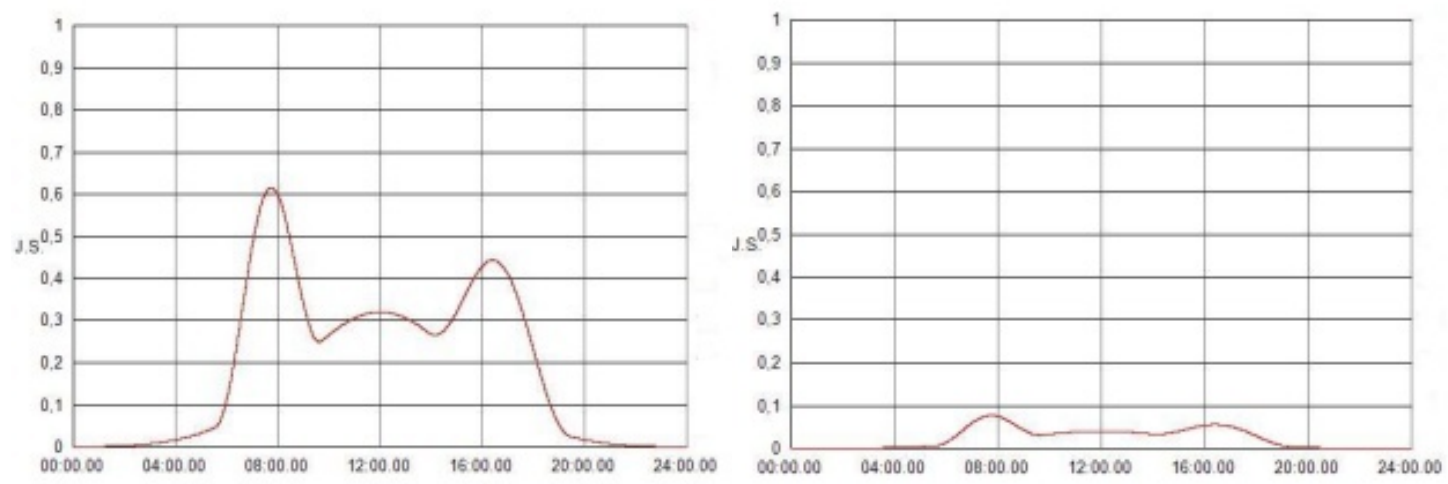

Fig. 9. Input function of prime - and side section

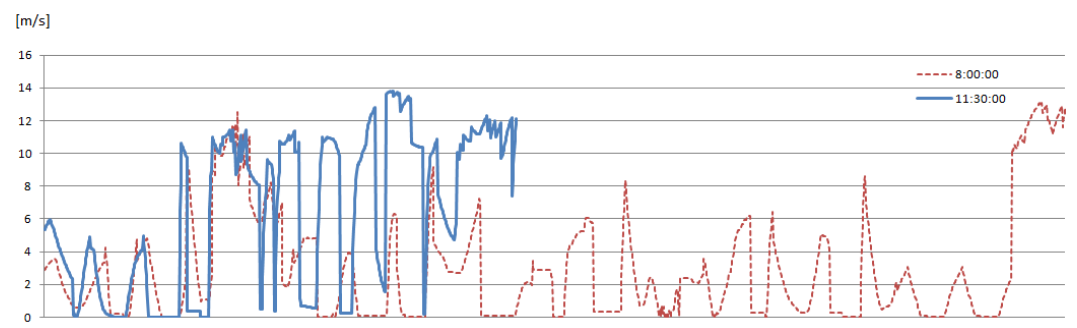

Fig. 10. Velocity diagrams in the morning peak (dashed line), concerning in a normal period (continuous line).

Before the starting of the simulation the certain elements of the edited network (input-, output-, and normal road lanes, and car parks) we may designate it for tracing [6]. The system makes an entry at this time, and it stores values of the taking shape vehicle density or velocity on the designate objects in the course of the simulation (according to selected in the window of a lane setting). Depending on setting during the simulation continuously, following his running down appears the graph of the first selected object in one block. We may save the single graphs, offering an opportunity to use in the course of the later simulations. Essential namely, that the effect of the completed changes can be analysed.
The feed data with the establishment of the traffic into the simulation are based on statistics. Like this, for example it $\alpha$ values, which mean the measure of the lane change, we granted it so, that the traffic is divided equally on the two lanes taking into consideration the intermediate exit and merge ramps.

\section{The comparison of velocity profiles}

Comparing some velocity profiles from the detailed traffic simulation with the standard procession cycles, conspicuous the principal deficiencies of the latter ones. The differences already noticeable from the velocity profile in case of values which can be counted directly, so for example the average speed and the 

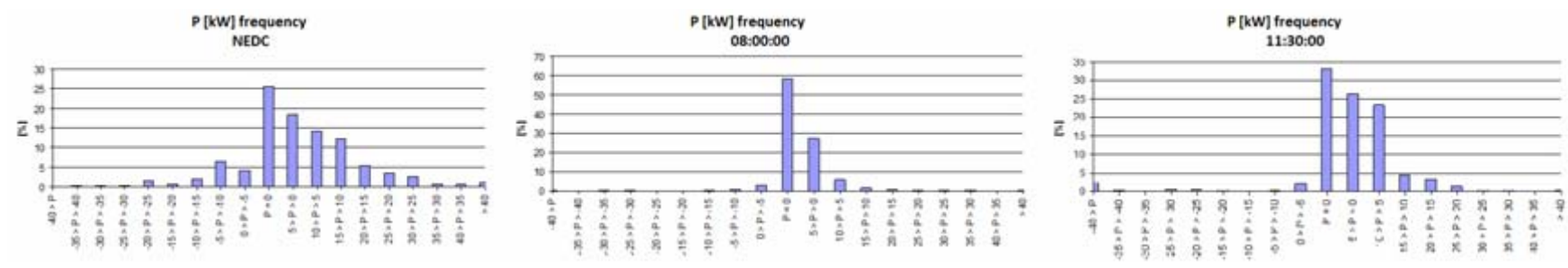

Fig. 11. The frequency of the performance claims in standard and procession cycles received with a traffic simulation

time in the position stop, in the neutral gear. From the given velocity values can be calculated the acceleration, and the tractive power depending on the instantaneous velocity and acceleration. The necessary performance to this we obtain in the knowledge of the strength to be created and the velocity. If compare performance claims belonging to the vehicles identical mass (2000 $\mathrm{kg}$ ) - and identical other features -, in the different procession cycles, we may see it, that on the standard NEDC cycle of the urban section the desired maximum $(\sim 20 \mathrm{~kW})$ is rest much under the value of a performance among the real circumstances desired $(\sim 31 \ldots 34 \mathrm{~kW})$. Of course the performance claim grows with the increase of the velocity on NEDC roadside section, however here it is not going above $50 \mathrm{~kW}$. The vehicle with a like performance and mass so, the pace of the urban traffic more or less tenable, although doubtless, that is not in possession of performance reserve. The peak values of the necessary braking performance exceed the obtain values in the standard cycle.

Examining the frequency of the performance claims on the Fig. 11. we may experience it, that in the standard cycle the distribution of the performance claim evener, in only about $25 \%$ we desire a most little performance around zero, while this proportion attains almost the $60 \%$ in the peak hours.

The significance of this consists in, that the $\mathrm{P}=0$ intervals signal the number of the stops and departures, so-called start-stop system may be effective here. Moreover at the time of the satisfaction of the most little performance claims the work dot of the internal combustion engine get into little load and low revolution, namely into the character field province with bad effective efficacy, so his specific consumption will be great. From here expedient to push away the work dot into a province with better efficacy, and the bigger performance of the engine to devote to charge of other deck energy containers - in case of a hybrid folding system the battery. The negative performance provinces mark the braking claim. Beside a negative performance claim it is most deprive the kinetic energy of the vehicle, which can be won back with suitable technology (recuperation). It is necessary to invest energy in positive performance intervals: such the phase of the acceleration or the phase of a keeping constant velocity. The performance diagrams mark two most important surface for us, where the efficiency of the folding chain is increasable. In a NEDC cycle in case of a hybrid-electric folding system the largest combustible saving can be reached with the offset of the work dot, follows this the recuperations energy and the application of the start-stop system.

\section{The validation of the model}

In the course of validation of model get to a comparison the velocity profiles (Fig. 12) as the result of the traffic simulation and fixed on the site as a function of the typical velocities of distribution and performance distribution. On this figure can be seen, that in the reality compared to the standard procession cycles much more the number of stops, sudden accelerations and intensive decelerations. We would like to compare these numbers with the velocity diagrams obtained from the simulation, to be proven the model's genuineness.

We did the route occasion three times with three different vehicles. We fixed the current position every second with the help of an apparatus GPS. We obtained the velocity profiles from these data, that we compared it with the velocity profiles obtained from the simulation. The comparison of these velocity profiles visible on the Fig.13

Longer examining the received results, we may see the velocity on the Fig. 14, concerning the performance distribution. In the course of the examination of the velocity distributions observable, that the values measured by the simulation disperse more steadily, than in the course of the real measurement. In both cases the value of velocity slow, $5 \mathrm{~km} / \mathrm{h}$ is most, according to the simulation this in an average is over $30 \%$, while according to GPS the average is around 50\%. Among the values with measured GPS are fewer the bigger of $30 \mathrm{~km} / \mathrm{h}$ velocity, than between the data obtained from the simulation. The simulation reckons with average speed on some sections and does not record bigger than the velocity permit, as this measurement take no notice of hindrance, for example the vehicles making parking along the route. If we compare the percent values all nine measured and simulated, then in the course of the velocity distribution the difference in an average $4,92 \%$ between the result of two kinds.

On the performance distributions the values originating from the measurement of two kinds stand even near to each other. An above mentioned the near zero performance values marking the stop and a departure in the course of all nine measurements, and all according to the simulation or GPS, in an average it is more than the $60 \%$ to compared to the overall performance claims. In the comparison of the valid percent the difference between the values got for the performance distribution are $1,65 \%$ in an average. 


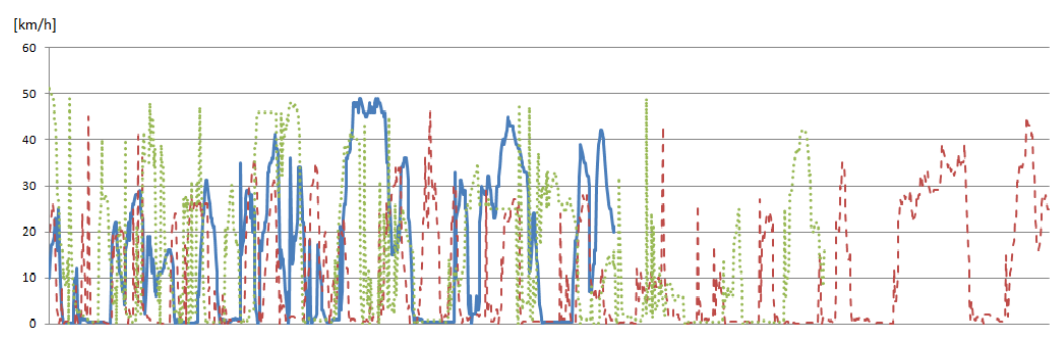

Fig. 12. Each vehicle measurement of velocity profiles obtained from an apparatus GPS

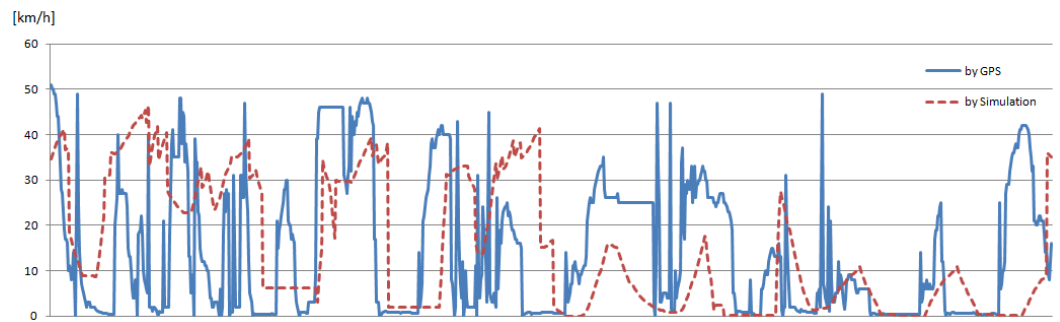

Fig. 13. Velocity profiles manufactured with a simulation and obtained from an apparatus GPS

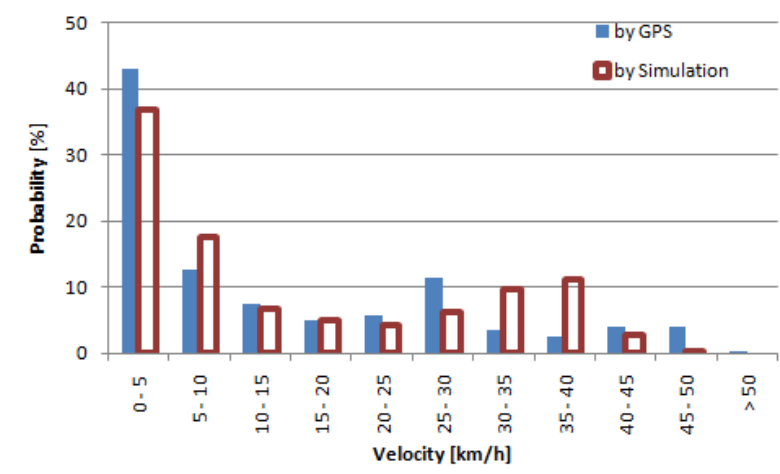

Fig. 14. The example of velocity and performance distribution

\section{Conclusion}

It by us created, the velocity distributions and performance distributions calculated from large urban network of road model are equal to the real, measured values. Our model calculated statistical values reflect sudden acceleration processes on the measured traffic typical, and according to the real processes, the durably permanently velocity values are not implied.

Based on this, proposed - the different from a NEDC cycle the application of above new velocity profiles onto the testing of the vehicles, that can be obtained data in the course of the test be much more accurate until now, which values are typical the vehicle of his real procession characteristics.

Our example in cases the measured and simulated distribution of velocities we measured a difference in an average $4,92 \%$, while the performance distribution altogether $1,65 \%$. So verifiable, that our model provides a suitable approach to the reality. The velocity profile suggested by us compared to procession cycles in the NEDC regulations, shows the real procession.

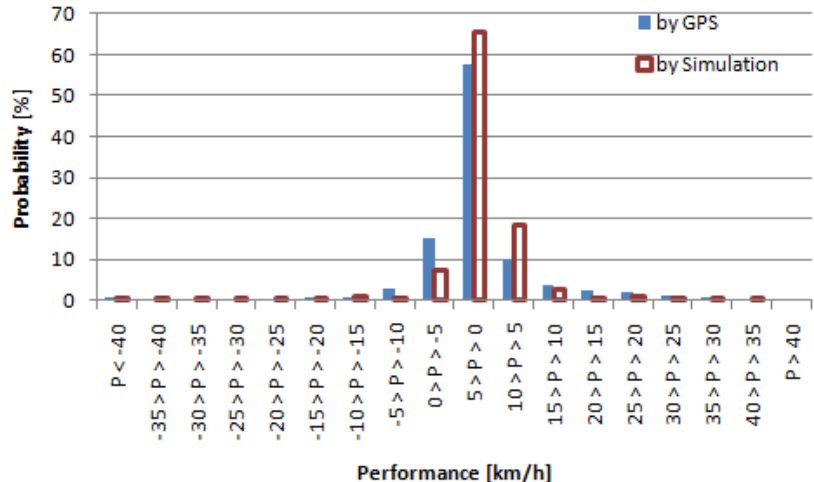

\section{References}

1 The technical analysis of alternative folding systems, Research meaning, BME Advanced Vehicle Control Knowledge Centre, Budapest, 2009.

2 Emöd I, Type examinations onto automobiles, BME Department of Automobile Engineering, Budapest, 2004.

3 , Official emission examinations, BME Department of Automobile Engineering, Budapest.

4 Blága Cs, Diagnostic of vehicle, ME Department of Electrical and Electronic Engineering, Miskolc, 2006.

5 Péter T, Stróbl A, Fazekas S, Software process analysis, large road traffic networks optimising, Vehicle of Future 2008, no. 1-2.

6 Péter T, Software development in BME: Onto large road traffic networks' analysis and his planning, IFFK Conference, 2008.

7 Péter $\mathbf{T}$, Bokor $\mathbf{J}$, The modelling of traffic systems and the research of his management, Vehicle of Future 2006, no. 1-2.

8 , For large road traffic networks' non-linear model hipermatrix of contact, Vehicle of Future 2007, no. 1-2. 